\title{
Fluorogel Elastomers with Tunable Transparency, Elasticity, Shape-Memory, and Antifouling Properties
}

\section{Citation}

Yao, Xi, Stuart S. Dunn, Philseok Kim, Meredith Duffy, Jack Alvarenga, and Joanna Aizenberg. 2014. Fluorogel Elastomers with Tunable Transparency, Elasticity, Shape-Memory, and Antifouling Properties. Angewandte Chemie International Edition 53, no. 17: 4418-4422. doi: 10.1002/anie.201310385

\section{Published Version}

doi:10.1002/anie.201310385

\section{Permanent link}

http://nrs.harvard.edu/urn-3:HUL.InstRepos:27662698

\section{Terms of Use}

This article was downloaded from Harvard University's DASH repository, and is made available under the terms and conditions applicable to Open Access Policy Articles, as set forth at http:// nrs.harvard.edu/urn-3:HUL.InstRepos:dash.current.terms-of-use\#OAP

\section{Share Your Story}

The Harvard community has made this article openly available.

Please share how this access benefits you. Submit a story.

Accessibility 


\title{
Fluorogel Elastomers with Tunable Transparency, Elasticity, Shape
Memory, and Antifouling Properties
}

\author{
Xi Yao*, Stuart S. Dunn, Philseok Kim, Meredith Duffy, Jack Alvarenga, and Joanna Aizenberg*
}

Developing materials and devices with tolerance to diverse operating conditions is fueled by technological needs. Optical lenses, flexible electronics and soft robotics require multifunctional materials with tunable optics, mechanics or actuation coupled with resistance to surface contamination ${ }^{[1-4]}$. Furthermore, biological implants such as catheters and artificial blood vessels need materials with controllable mechanics while exhibiting sustained biocompatibility and anti-biofouling properties to prevent infection. Exemplary technologies harness multi-component systems with specific chemical moieties to tune surface chemistry ${ }^{[5-10]}$. However, such multi-component systems create excessive complexity that often sacrifices properties, and cannot fully avoid the function loss resulting from surface contamination. Moreover, the molecular mechanisms that prevent liquid contamination are generally different from those involved in prevention of biofouling, which makes integration of both functions in one material fundamentally difficult $^{[11-14]}$. For example, hydrophilic coatings such as poly(ethylene glycol) (PEG) are often used for the prevention of non-specific bio-adhesion, while hydrophobic surfaces are usually required for liquid repellency. Here we develop a family of multifunctional, omniphobic perfluorinated elastomers that combine exceptional liquid repellency with tunable optics, mechanics and shape-memory. By tuning either chemical composition or processing temperature that controls the crystalline status of the of polymer chains, a broad range of optical and mechanical properties can be achieved. Fluorinated lubricants are infused into the perfluorinated elastomers to yield a new type of organogels, namely "fluorogels", which demonstrate broad anti-fouling against water, hydrocarbon oils, proteins, cells and blood while maintaining excellent cytocompatibility.

Fluorogels were designed from select perfluorinated alkyl acrylate monomers and a fluorinated macromolecular crosslinker. Specifically, 2-perfluorooctylethyl acrylate (PFOEA) or 2perfluorohexylethyl acrylate (PFHEA) were mixed with perfluoropolyether dimethacrylate (PFPE-DMA) in different ratios to yield fluorogels (naming convention: monomer-volume\%). It was envisioned that the morphology of the fluorogel polymer network

[*] (Dr. X. Yao+, Dr. S. Dunn+. Dr. P. Kim, M. Duffy, J. Alvarenga, Prof. J. Aizenberg

School of Engineering and Applied Sciences

Wyss Institute for Biologically Inspired Engineering

Harvard University

29 Oxford Street, Cambridge, MA 02138 (USA)

E-mail: yaoxi@seas.harvard.edu; jaiz@seas.harvard.edu

[+] These authors contributed equally to this work.

[**] We thank Dr. M. Aizenberg for discussions. This work was supported by the Advanced Research Projects AgencyEnergy (ARPA-E) under award number DE-AR0000326.

Supporting information for this article is available on the WWW under http://www.angewandte.org or from the author. could be precisely tuned from semicrystalline to amorphous by specifying the identity and/or amount of monomer in the matrix ${ }^{[15-17]}$ As evidenced by differential scanning calorimetry (DSC) (Figure 1a), melting and crystallization phase transitions were noted only for PFOEA-based gels with monomer content larger than $50 \%$ as enthalpy changes were not observed on PFHEA-based gels between -70 and $150^{\circ} \mathrm{C}$. The length of the fluorinated side chain of PFOEA is sufficient for crystallization while that of PFHEA does not provide crystallization ${ }^{[15,16]}$. The magnitude of the change in heat flow decreased with fluorogels from PFOEA-100 to PFOEA-50, which suggests that the amount of PFOEA side chains that are available for packing into semi-crystalline domains decreases with increasing crosslinker content, which is also further evidenced by atomic force microscopy (Figure 1b, Figure S1). Additionally, robust thermal stability was observed for these fluorogels as determined by thermal gravimetric analysis; $0.5 \%$ total mass loss for PFOEA-50 at $134^{\circ} \mathrm{C}, 2 \%$ total mass loss at $265^{\circ} \mathrm{C}$, and $50 \%$ total mass loss occurred around $375^{\circ} \mathrm{C}$, respectively (Figure S2).

(a)

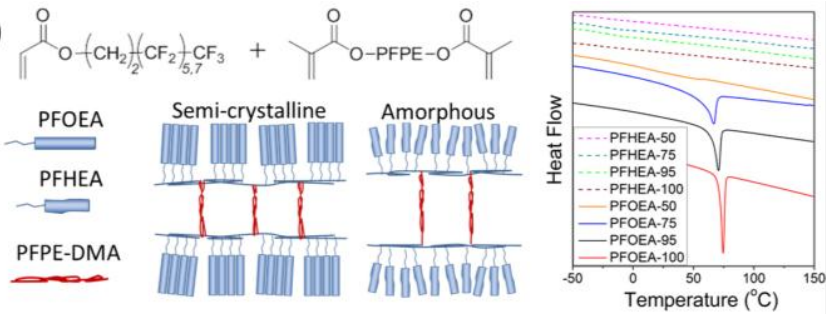

(b)

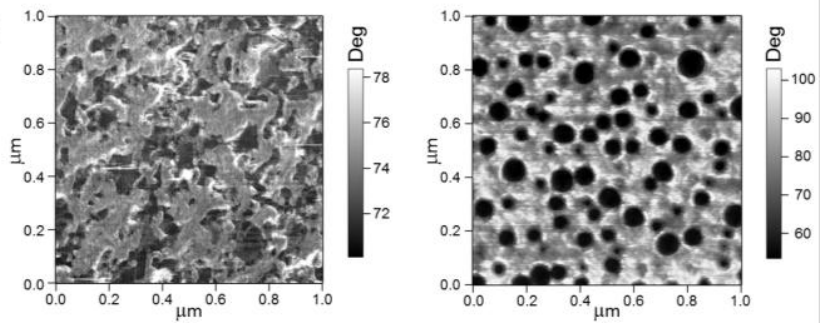

Figure 1. (a) Chemical design of fluorogels, molecular configuration of side chains, and DSC analysis of fluorogels. (b) Tapping mode phase AFM images of PFOEA-100 (left) and PFOEA-50 (right) fluorogel film prepared on glass slides, indicating that the surface of PFOEA-100 films is notably more homogeneous than that of PFOEA50. Domains of different softness were observed in PFOEA-50, which may be ascribed to semicrystalline and amorphous regions from the PFOEA side chain and PFPE crosslinker, respectively.

Defining the morphology of the fluoropolymer network allows for control over the physical properties and corresponding behavior of the material. The transparency ranged from opaque to transparent, and the mechanical properties extended from a stiff, rigid plastic to a soft elastomer with the modulus spanning three orders of magnitude (Table S1). The PFOEA-100 samples were rigid (E $200 \mathrm{MPa}$ ) and opaque (T\% 0\%) where the large crystalline domains contributed to light scattering and high modulus. PFOEAbased networks became increasingly flexible, soft, and transparent with increasing content of crosslinker, which breaks up the 
semicrystalline domains. PFHEA-based samples were all clear and soft where modulus depended inversely on monomer content due to their amorphous nature and short-chain perfluoroalkyl monomer reaching $\sim 0.1 \mathrm{MPa}$ (Figure 2a-b).

Thermally induced crystalline-amorphous transition enabled tunable optical and mechanical properties for fluorogels with notable semicrystallinity. For example, a film of PFOEA-95 turned transparent when it was heated above $70^{\circ} \mathrm{C}$, and reverted back to opaque after cooling down to $25^{\circ} \mathrm{C}$ (Figure 2c,i-ii and Figure S3). Crosslinked polymers with high degrees of crystallinity have been shown to exhibit thermally-responsive shape memory behavior ${ }^{[18,19]}$. Heating semicrystalline fluorogel, such as PFOEA-95, above its transition temperature resulted in soft and processable materials that retained any shape after cooling down. After re-heating above the transition temperature, the fluorogel recovered its initial shape due to the covalent crosslinks (Figure 2c).

(a)
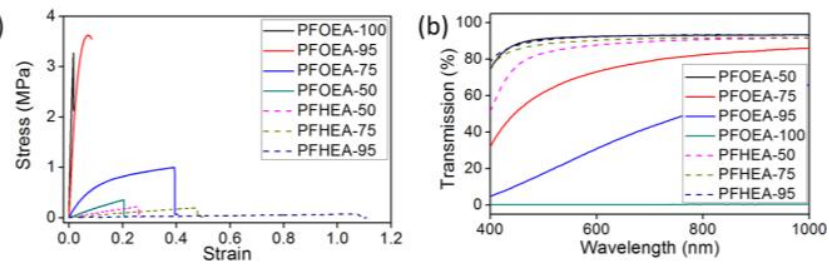

(c)

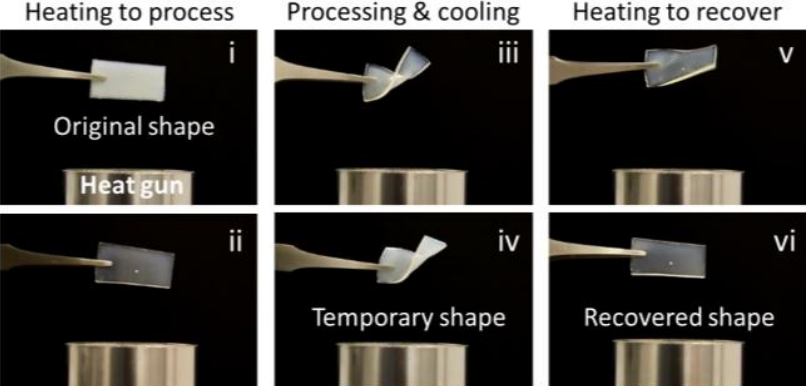

Figure 2. Tunable mechanical and optical properties of fluorogels. (a) Tensile and (b) optical tests for fluorogels of different compositions. Samples of $1 \mathrm{~mm}$ thickness were prepared for all the optical measurements. (c) Demonstration of the shape memory behavior of PFOEA-95: i-ii, Converting a rigid gel film (white) to a soft and flexible (transparent) film upon heating with a heat gun; iii-iv, Twisting a soft gel film and keeping the shape when cooling down; v-vi, Recovering the original shape upon heating.

The non-wetting properties of the fluorogels were exemplified by superior chemical resistance to most organic solvents such as pentane, hexane, toluene, ethanol, etc. (Table S2). As a class of fluorinated elastomers, fluorogels were subsequently investigated for their swelling properties. Fluorogels were slightly swollen by some halogenated solvents such as chloroform or trifluoroethanol, and showed great affinity to fluorinated solvents such as FC-70 and DuPont Krytox oils (Figure S4), which are commonly used as lubricants in liquid-infused materials to achieve interfacial slipperiness and universal repellency due to their immiscibility with most other liquids ${ }^{[20,21]}$. Several days were required for the fluorogels to reach equilibrium swelling, and larger extents of swelling were noted with FC-70, a small molecule, compared to Krytox 100, a macromolecule (Figure 3a-b). Furthermore, the magnitude of swelling increased with decreasing modulus for fluorogels swollen by FC-70 as swelling is promoted by mixing the polymer with solvent and is discouraged by the stretching of network strands ${ }^{[22]}$.
These unique swelling and chemical resistance abilities make fluorogels suitable as matrices for liquid-infused materials. The bare fluorogels are omniphobic, on which droplets of water or hexadecane, with contact angles of ca. $120^{\circ}$ and $70^{\circ}$ (Figure S5), respectively, did not spread but slid away upon tilting of the substrates. The contact angle hysteresis values were relatively high, i.e., $40-55^{\circ}$ for hexadecane and $33-48^{\circ}$ for water on bare PFOEAbased gels. Although several days may be required to completely swell fluorogels, material properties were evaluated after $24 \mathrm{~h}$ of lubricant infusion to minimize the time and amount of lubricant required for applications. With an overcoated lubricant layer, swollen fluorogels exhibited slippery properties with water or hexadecane sliding angles less than $10^{\circ}$. To decouple the effect of the thick lubricant layer from the swollen gel, the surface was wiped dry to remove the excess lubricant layer after swelling. As shown in Figure $3 c-d$, the contact angle hysteresis of water or hexadecane decreased down to around $15^{\circ}-20^{\circ}$ for PFOEA-based fluorogels after swelling in FC-70 for $24 \mathrm{~h}$. Similar results were observed when the swelling liquid changed to Krytox 100, and for PFHEA-based fluorogels.

As an alternative to swelling fluorogels post fabrication, lubricant may be incorporated into the fluorogel through a one-pot method by adding the lubricant to the precursor solution. Water and hexadecane contact angle hysteresis decreased with increasing Krytox lubricant concentration, reaching less than $10^{\circ}$ at a lubricant:precursor ratio of 4:1 (volume) (Figure 3e, Figure S6). Fluorogels prepared by either post fabrication swelling or the onepot method exhibited viscoelastic rheological properties, and the modulus decreased with increasing concentration of lubricant (Figure S7, S8).
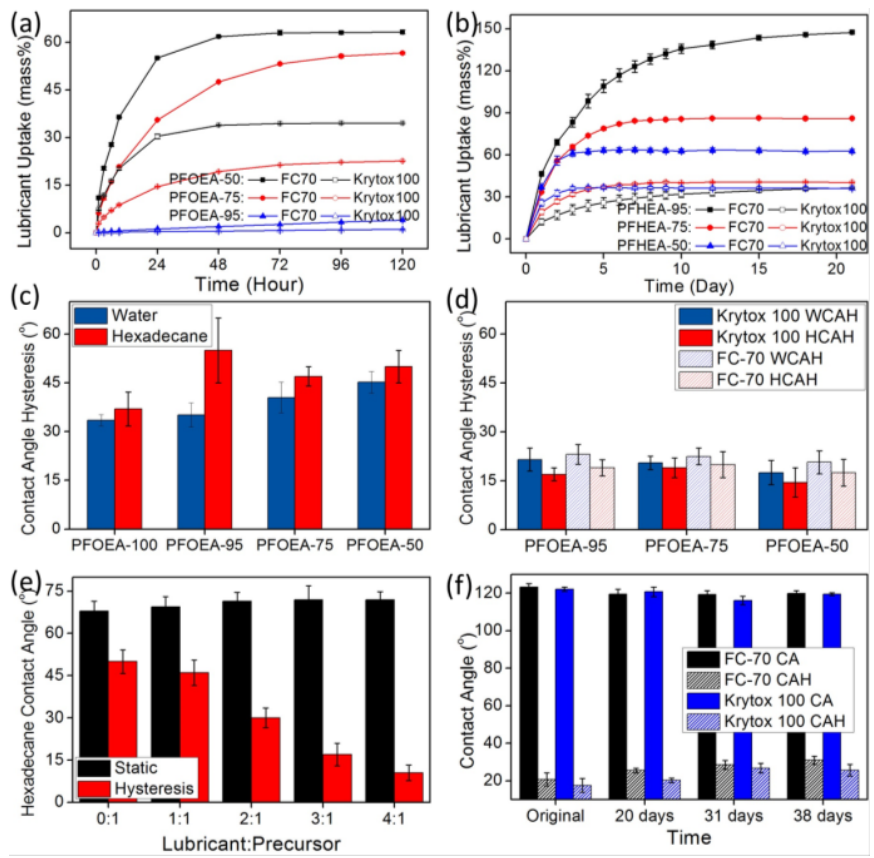

Figure 3. (a-b) Mass uptake of different lubricants and timedependent swelling dynamics for PFOEA-50 (a) and PFHEA-95 (b) fluorogels. (c-f) Wetting properties of PFOEA-based fluorogels: (c) contact angle hysteresis of water (WCAH) and hexadecane (HCAH) on bare fluorogel; (d) WCAH and HCAH on lubricant-swollen fluorogels; (e) Contact angle hysteresis of hexadecane on fluorogels prepared via one-pot method, specifically, PFOEA-50 mixed with FC70 in different volume ratios; (f) Contact angle (CA) and contact angle hysteresis $(\mathrm{CAH})$ of water on lubricant-swollen PFOEA-50 fluorogels incubated under ambient conditions at different time points. 
The crosslinked network of fluorogels may retard the loss of encapsulated lubricant in the matrix and maintain function for a long time even if the lubricant is relatively volatile, as in the case of FC70. Contact angle hysteresis of water remained approximately $25-30^{\circ}$ and $20-25^{\circ}$ for FC-70 and Krytox 100 swollen samples, respectively, after more than a month (Figure $3 \mathrm{f}$ ). As there is no apparent change in topography for fluorogels during the swelling process nor excess lubricating layer present after wiping samples dry, the slippery properties and the extended longevity may be attributed to swollen gel interface coated with the lubricant layer that is constantly replenished at the gel surface due to directional diffusion (Figure 4a).

(a)
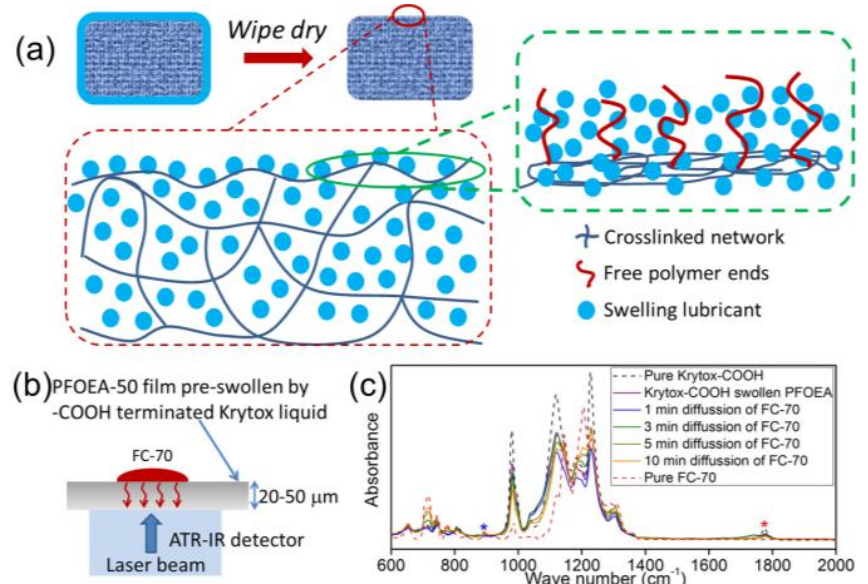

(d)

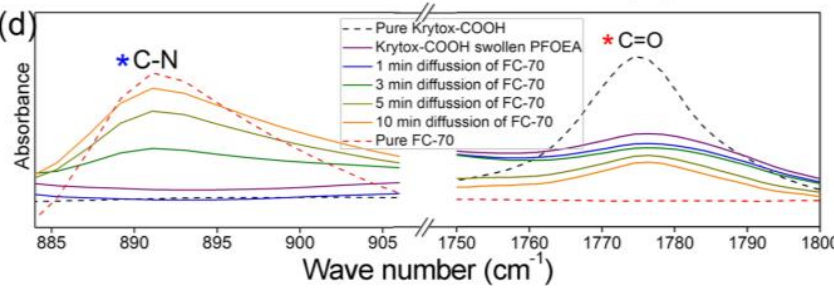

Figure 4. (a) Schematic llustration of the presence of lubricant dispersed throughout the polymer network and diffusing to the polymer surface, with free polymer chains at the swollen gel surface that reduce friction and provide slipperiness. (b-d) Analysis of lubricant diffusion in fluorogels by ATR-IR: (b) Schematic of experiment setup, (c) Full spectrum, (d) C-N (blue star) (left) and $\mathrm{C}=\mathrm{O}$ (red star) (right) stretch peaks from ATR-IR analysis of pure FC70, pure Krytox-COOH, Krytox-COOH swollen PFOEA-50, and the Krytox- $\mathrm{COOH}$ swollen PFOEA-50 with FC-70 diffusion at 1, 3, 5 and $10 \mathrm{~min}$. The increase of $\mathrm{C}-\mathrm{N}$ signal and the decrese of $\mathrm{C}=\mathrm{O}$ signal with increasing diffusion time imply that the Krytox- $\mathrm{COOH}$ was diluted or even replaced by the diffusion of FC-70.

We anticipate that the swelling mechanism involves a homogeneous, molecular-level infusion of lubricant, resulting in a wet, lubricated environment at the gel surface ${ }^{[23]}$. Free, dangling polymer chain ends together with the wet environment can help reduce interfacial friction and thus promote slipperiness of the surface $^{[24-26]}$. The presence of this lubricating layer on the surface was confirmed by the Attenuated Total Reflectance-Infrared spectroscopy (ATR-IR). As shown in Figure 4b-d, a thin film (20-50 $\mu \mathrm{m})$ of PFOEA-50 was swollen with - $\mathrm{COOH}$-terminated Krytox oligomer (DuPont, M.W. 2500), wiped dry and placed onto the detector of ATR-IR. In situ ATR-IR measurements were carried out when a drop $(10 \mu \mathrm{L})$ of FC-70 was deposited onto the upper surface of the swollen fluorogel sample. Increasing signal of $\mathrm{C}-\mathrm{N}$ bond stretch was observed with increasing diffusion time, showing that FC-70 indeed diffused into and reached the other side of the pre- swollen sample. The maintenance of FC-70 at the swollen gel surface (top few microns) was noted up to five days even when the gel was placed under vacuum (Figure S9).

The surface of fluorogels can also be patterned at the nano and micron scale into any desired topography using soft lithography to generate superhydrophobicity or even slipperiness with lubrication (Figure S10).

Since the swollen fluorogels show omniphobicity and slipperiness, anti-biofouling properties were subsequently studied. Biofouling of surfaces can negatively affect the performance of medical devices and sensitivity of diagnostics ${ }^{[27]}$. To evaluate the biological and anti-biofouling activity of fluorogels, their interaction with proteins, cells, and blood was assessed. Reduced protein adsorption was noted on both bare and swollen fluorogels relative to a positive control (cationic hydrogels), and fluorogels performed similarly to an anti-biofouling surface benchmark (PEG hydrogels), as indicated by confocal fluorescence spectroscopy (Fig. 5a). A closer examination of the protein adsorption among fluorogel samples through spectrophotometric analysis showed that both FC70-swollen bulk and patterned fluorogel did not display the presence of protein while bare fluorogels showed notable protein adsorption. The interaction of biological cells with fluorogels was studied for cytocompatibility (Figure S11) and adhesion/spreading properties (Fig. 5c). The control (tissue culture polystyrene) enabled good adhesion and spreading of cells while ca. four-fold smaller area per cell was noted after incubation with fluorogels, indicating that they were mostly unattached, with complete viability. Next, blood bank blood was dispensed on bulk material of FC-70-swollen or bare PFOEA-50 fluorogels. The ability of materials to minimize adhesion of blood to their surface and prevent coagulation finds importance in several medical applications such as dialysis, transfusion, analyte detection, and pathogen removal. Only swollen fluorogels repelled blood, while pinning and streaking of blood on bare fluorogels was observed, which can cause undesired coagulation (Figure 5d).

In conclusion, lubricant-infused fluorogels are a unique, modular and customizable polymeric gel system possessing omniphobicity, slipperiness, broad anti-biofouling, tunable mechanical and optical properties, and a shape memory behavior. Our study provides a general framework for the design of lubricant-infused polymeric gels with controlled properties and functions that should be amenable to a variety of applications. We anticipate that these multifunctional fluorogels can offer unforeseen combinations of tunable properties and anti-fouling performance, which will enable new technologies and improve performance/efficiency in a range of energy, environmental, and biomedical applications that require long-term operations and/or encounter harsh environmental conditions. Due to its modularity, the family of fluorogels may be expanded by selecting monomers and crosslinkers with different structures, or implementing additives such as fillers or functional monomers. The flexibility and chemical simplicity of fluorogels may enable integration with a wide range of materials, response to different stimuli, and design of unique behaviors. Both the lubricant and polymer network can be rationally chosen for specific crystalline, optical, swelling and slippery properties to meet the requirements for a particular application and/or environment.

Received: ((will be filled in by the editorial staff))

Published online on ((will be filled in by the editorial staff $)$ )

Keywords: polymer gel · omniphobic surface $\cdot$ slippery surface . shape memory · anti-biofouling 


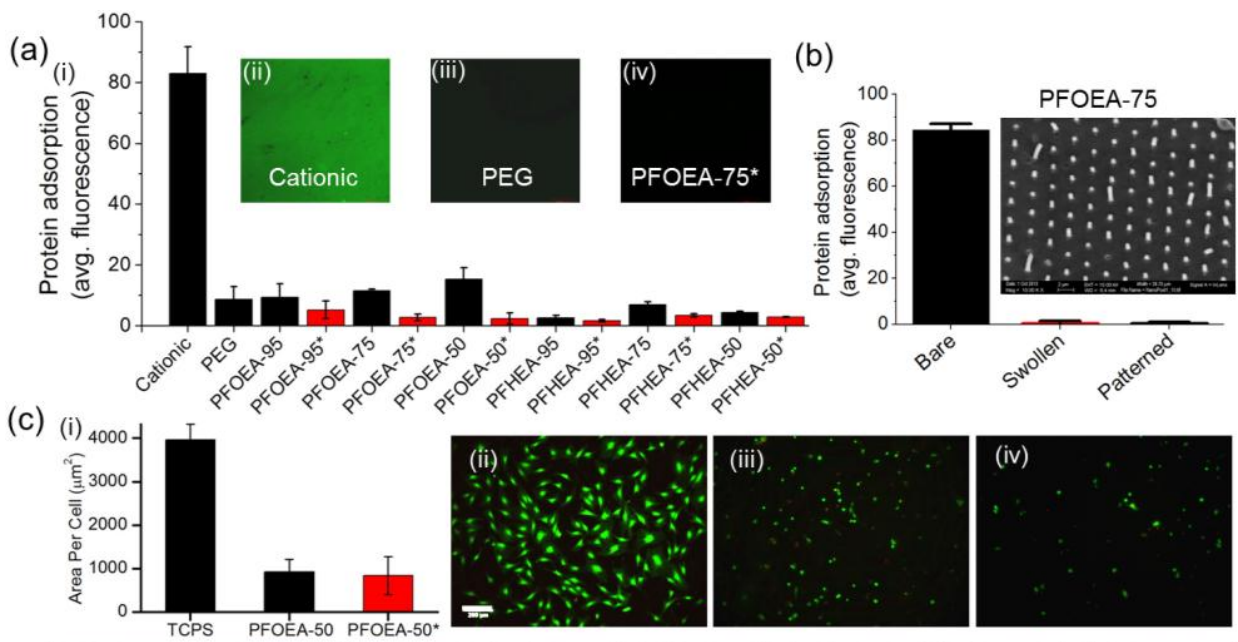

(d)

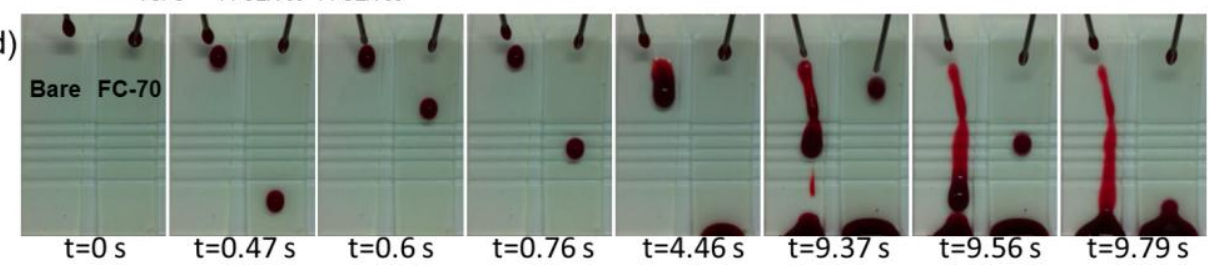

Figure 5. Anti-biofouling behavior of fluorogels (swollen gels are indicated by asterisk and marked as red column). (a) Protein adhesion to different fluorogels: (i) Average fluorescence intensity of samples exposed to fluorescently tagged BSA protein; (ii)-(iv) confocal micrographs of (ii) cationic hydrogels, (iii) PEG hydrogels, and (iv) swollen PFHEA-75 samples after incubation with protein; (b) spectrophotometric analysis of fluorogels (bare, FC-70swollen, and nanopost-patterned, FC70-swollen PFOEA-75) exposed to protein. Inset shows an SEM image of the patterned fluorogel. (c) Assessing adhesion of mouse embryonic fibroblasts to different substrates: (i) Quantification of cell spreading on substrates; (ii)-(iv) fluorescent images of samples after incubation with cells for $24 \mathrm{~h}$ on (ii) tissue culture polystyrene, (iii) bare PFOEA-50 fluorogel, and (iv) FC-70-swollen fluorogel. (d) Application of blood to bare and FC-70-swollen PFOEA-50 fluorogels: time lapse images show blood sliding on swollen fluorogels and pinning and streaking on bare fluorogels.

[1] R. Langer, D. A. Tirrell, Nature 2004, 428, 487.

[2] I. Banerjee, R. C. Pangule, R. S. Kane, Adv. Mater. 2011, 23, 690.

[3] D. V. Manov, G. C. Chang, T. D. Dickey, J. Atmos. Ocean. Tech. 2004, 21, 958

[4] R. V. Shevchenko, S. L. James, S. E. James, Journal of the Royal Society Interface 2010, 7, 229.

[5] A. Sethuraman, M. Han, R. S. Kane, G. Belfort, Langmuir 2004, 20, 7779 .

[6] K. N. Ren, W. Dai, J. H. Zhou, J. Su, H. K. Wu, Proceedings of the National Academy of Sciences of the United States of America 2011, 108, 8162.

[7] N. A. Peppas, J. Z. Hilt, A. Khademhosseini, R. Langer, Adv. Mater. 2006, 18, 1345.

[8] J. L. Drury, D. J. Mooney, Biomaterials. 2003, 24, 4337.

[9] A. Vintiloiu, J. C. Leroux, J. Control Release. 2008, 125, 179.

[10] T. Aida, E. W. Meijer, S. I. Stupp, Science 2012, 335, 813.

[11] J. P. Rolland, R. M. Van Dam, D. A. Schorzman, S. R. Quake, J. M. DeSimone, J. Am. Chem. Soc. 2004, 126, 2322.

[12] Z. K. Hu, L. Chen, D. E. Betts, A. Pandya, M. A. Hillmyer, J. M. DeSimone, J. Am. Chem. Soc. 2008, 130, 14244.

[13] Y. P. Wang, D. E. Betts, J. A. Finlay, L. Brewer, M. E. Callow, J. A. Callow, D. E. Wendt, J. M. DeSimone, Macromolecules 2011, 44, 878.

[14] C. S. Gudipati, J. A. Finlay, J. A. Callow, M. E. Callow, K. L. Wooley, Langmuir 2005, 21, 3044.

[15] J. M. Corpart, S. Girault, D. Juhue, Langmuir 2001, 17, 7237.

[16] K. Honda, M. Morita, H. Otsuka, A. Takahara, Macromolecules 2005, 38, 5699.

[17] T. Nishino, Y. Urushihara, M. Meguro, K. Nakamae, J. Colloid Interf. Sci 2005, 283, 533.

[18] I. A. Rousseau, Polym. Eng. Sci. 2008, 48, 2075.
[19] P. T. Mather, X. F. Luo, I. A. Rousseau, Ann. Rev. Mater. Res. 2009, 39, 445.

[20] X. Yao, Y. H. Hu, A. Grinthal, T. S. Wong, L. Mahadevan, J. Aizenberg, Nat. Mater. 2013, 12, 529.

[21] T. S. Wong, S. H. Kang, S. K. Y. Tang, E. J. Smythe, B. D. Hatton, A. Grinthal, J. Aizenberg, Nature 2011, 477, 443.

[22] M. Rubinstein, R. H. Colby, Polymer Physics, Oxford University Press, New York, NY 2007.

[23] Y. Osada, J. P. Gong, Adv. Mater. 1998, 10, 827.

[24] J. P. Gong, Soft Matter 2006, 2, 544.

[25] A. Li, E. M. Benetti, D. Tranchida, J. N. Clasohm, H. Schonherr, N. D. Spencer, Macromolecules 2011, 44, 5344.

[26] M. Sitti, B. Cusick, B. Aksak, A. Nese, H. I. Lee, H. C. Dong, T. Kowalewski, K. Matyjaszewski, Acs Applied Materials \& Interfaces 2009, 1, 2277.

[27] A. Hucknall, S. Rangarajan, A. Chilkoti, Adv. Mater. 2009, 21, 2441. 
Entry for the Table of Contents (Please choose one layout)

\section{Polymeric Gels}

Xi Yao, Stuart S. Dunn, Philseok Kim, Meredith Duffy, Jack Alvarenga, and Joanna Aizenberg* Page -

Page

Fluorogel Elastomers with Tunable Transparency, Elasticity, Shape Memory, and Anti-biofouling Properties
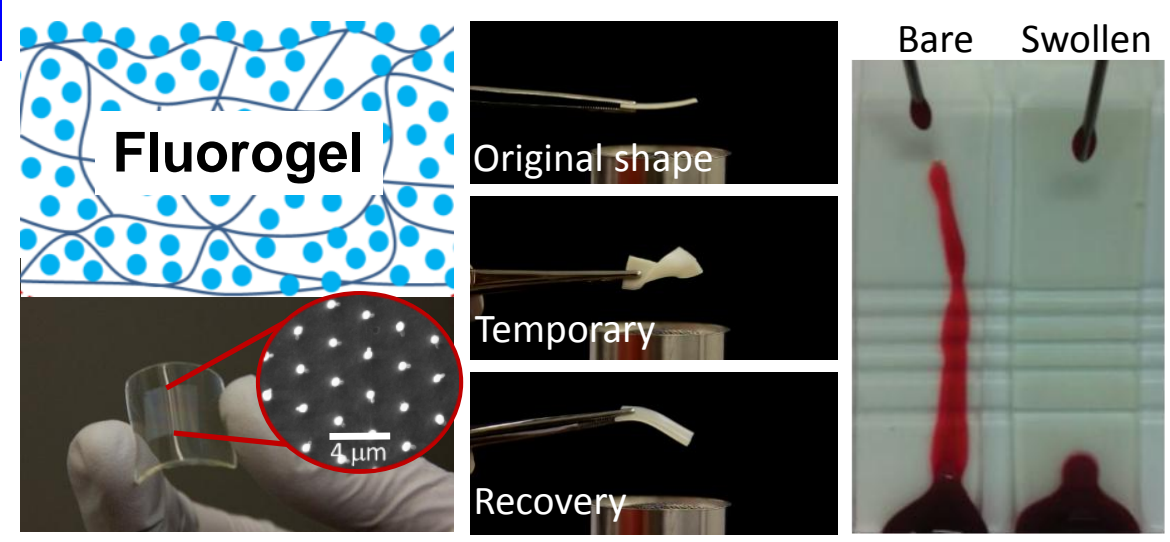

Omniphobic fluorogel elastomers were prepared by photocuring perfluorinated acrylates and a perfluoropolyether crosslinker. The optical transparency, mechanics, and shape memory behavior of fluorogels could be controlled by tuning the morphology from semicrystalline to amorphous. After infusing fluorinated lubricants into the perfluorinated elastomers, fluorogels demonstrated broad antifouling against water, hydrocarbon oils, proteins, cells and blood while maintaining excellent cytocompatibility. 